\title{
Evaluating Multiple Integrals Using Maple
}

\author{
Chii-Huei Yu ${ }^{1, *}$, Bing-Huei Chen ${ }^{2}$ \\ ${ }^{1}$ Department of Management and Information, Nan Jeon University of Science and Technology, Tainan City, 73746, Taiwan \\ ${ }^{2}$ Department of Electrical Engineering, Nan Jeon University of Science and Technology, Tainan City, 73746, Taiwan \\ *Corresponding Author: chiihuei@mail.nju.edu.tw
}

Copyright (C) 2014 Horizon Research Publishing All rights reserved.

\begin{abstract}
This paper uses the mathematical software Maple for the auxiliary tool to study two types of multiple integrals. We can obtain the infinite series forms of these two types of multiple integrals by using binomial series and integration term by term theorem. On the other hand, we propose some examples to do calculation practically. The research methods adopted in this study involved finding solutions through manual calculations and verifying these solutions by using Maple.
\end{abstract}

Keywords Multiple Integrals, Infinite Series Forms, Binomial Series, Integration Term By Term Theorem, Maple

\section{Introduction}

As information technology advances, whether computers can become comparable with human brains to perform abstract tasks, such as abstract art similar to the paintings of Picasso and musical compositions similar to those of Mozart, is a natural question. Currently, this appears unattainable. In addition, whether computers can solve abstract and difficult mathematical problems and develop abstract mathematical theories such as those of mathematicians also appears unfeasible. Nevertheless, in seeking for alternatives, we can study what assistance mathematical software can provide. This study introduces how to conduct mathematical research using the mathematical software Maple. The main reasons of using Maple in this study are its simple instructions and ease of use, which enable beginners to learn the operating techniques in a short period. By employing the powerful computing capabilities of Maple, difficult problems can be easily solved. Even when Maple cannot determine the solution, problem-solving hints can be identified and inferred from the approximate values calculated and solutions to similar problems, as determined by Maple. For this reason, Maple can provide insights into scientific research. Inquiring through an online support system provided by Maple or browsing the Maple website (www.maplesoft.com) can facilitate further understanding of Maple and might provide unexpected insights. For the instructions and operations of Maple, [1-7] can be adopted as references.

The multiple integral problem is closely related with probability theory and quantum field theory, and can refer to [8-9]. For this reason, the evaluation and numerical calculation of multiple integrals is important. In this paper, we mainly study the following two types of $n$-tuple integrals

$$
\begin{aligned}
& \int_{t_{n}}^{r_{n}} \cdots \int_{t_{1}}^{r_{1}} \exp \left(\sum_{k=1}^{n} a_{k} x_{k}\right) \cosh ^{p}\left(\sum_{k=1}^{n} b_{k} x_{k}\right) d x_{1} \cdots d x_{n}(1) \\
& \int_{t_{n}}^{r_{n}} \cdots \int_{t_{1}}^{r_{1}} \exp \left(\sum_{k=1}^{n} a_{k} x_{k}\right) \sinh ^{p}\left(\sum_{k=1}^{n} b_{k} x_{k}\right) d x_{1} \cdots d x_{n} \text { (2) }
\end{aligned}
$$

Where $n$ is any positive integer, $p, a_{k}, b_{k}, r_{k}, t_{k}$ are real numbers for all $k=1, \ldots, n$. We can obtain the infinite series forms of these two types of multiple integrals by using binomial series and integration term by term theorem; these are the major results of this study (i.e., Theorems 1 and 2). Moreover, we obtain some corollaries from these two theorems. For the study of related multiple integral problems can refer to [10-23]. In addition, we provide some multiple integrals to do calculation practically. The research methods adopted in this study involved finding solutions through manual calculations and verifying these solutions by using Maple. This type of research method not only allows the discovery of calculation errors, but also helps modify the original directions of thinking from manual and Maple calculations. Therefore, Maple provides insights and guidance regarding problem-solving methods.

\section{Main Results}

Firstly, we introduce two notations and two important theorems used in this paper.

\subsection{Notations}

2.1.1. $\prod_{k=1}^{n} \lambda_{k}=\lambda_{1} \times \lambda_{2} \times \cdots \times \lambda_{n}$, where $n$ is a positive integer, and $\lambda_{k}$ are real numbers for all $k=1, . ., n$.

2.1.2. Suppose $r$ is any real number, $m$ is any positive 
integer. Define $(r)_{m}=r(r-1) \cdots(r-m+1)$, and $(r)_{0}=1$.

\subsection{Binomial series ([24])}

$(1+y)^{r}=\sum_{m=0}^{\infty} \frac{(r)_{m}}{m !} y^{m}$, where $y, r$ are real numbers, $|y|<1$.

\subsection{Integration term by term theorem ([25])}

Suppose $\left\{g_{n}\right\}_{n=0}^{\infty}$ is a sequence of Lebesgue integrable functions defined on an interval $I$. If $\sum_{n=0}^{\infty} \int_{I}\left|g_{n}\right|$ is convergent, then $\int_{I} \sum_{n=0}^{\infty} g_{n}=\sum_{n=0}^{\infty} \int_{I} g_{n}$.

The following is the first result in this study, we find the infinite series forms of the multiple integral (1).

2.4. Theorem 1 Suppose $n$ is any positive integer, and $p, a_{k}, b_{k}, r_{k}, t_{k}$ are real numbers, $b_{k} \neq 0$ for all $k=1, . ., n$.

Case (A). If $\sum_{k=1}^{n} b_{k} x_{k}>0$, and $\frac{a_{k}+p b_{k}}{2 b_{k}}$ are not non-negative integers for all $k=1, . ., n$. Then the $n$ -tuple integral

$$
\begin{aligned}
& \int_{t_{n}}^{r_{n}} \cdots \int_{t_{1}}^{r_{1}} \exp \left(\sum_{k=1}^{n} a_{k} x_{k}\right) \cosh ^{p}\left(\sum_{k=1}^{n} b_{k} x_{k}\right) d x_{1} \cdots d x_{n} \\
= & \frac{1}{2^{p}} \cdot \sum_{m=0}^{\infty} \frac{(p)_{m} \cdot \prod_{k=1}^{n}\left\{\exp \left[a_{k}+(p-2 m) b_{k}\right] r_{k}-\exp \left[a_{k}+(p-2 m) b_{k}\right] t_{k}\right\}}{m ! \cdot \prod_{k=1}^{n}\left[a_{k}+(p-2 m) b_{k}\right]}
\end{aligned}
$$

Case (B). If $\sum_{k=1}^{n} b_{k} x_{k}<0$, and $\frac{a_{k}-p b_{k}}{-2 b_{k}}$ are not non-negative integers for all $k=1, . ., n$. Then

$$
\begin{aligned}
& \int_{t_{n}}^{r_{n}} \cdots \int_{t_{1}}^{r_{1}} \exp \left(\sum_{k=1}^{n} a_{k} x_{k}\right) \cosh ^{p}\left(\sum_{k=1}^{n} b_{k} x_{k}\right) d x_{1} \cdots d x_{n} \\
= & \frac{1}{2^{p}} \cdot \sum_{m=0}^{\infty} \frac{(p)_{m} \cdot \prod_{k=1}^{n}\left\{\exp \left[a_{k}-(p-2 m) b_{k}\right] r_{k}-\exp \left[a_{k}-(p-2 m) b_{k}\right] t_{k}\right\}}{m ! \prod_{k=1}^{n}\left[a_{k}-(p-2 m) b_{k}\right]}
\end{aligned}
$$

2.4.1. Proof Case (A). If $\sum_{k=1}^{n} b_{k} x_{k}>0$ and $\frac{a_{k}+p b_{k}}{2 b_{k}}$ are not non-negative integers for all $k=1, . ., n$. Because

$$
\exp \left(\sum_{k=1}^{n} a_{k} x_{k}\right) \cosh ^{p}\left(\sum_{k=1}^{n} b_{k} x_{k}\right)
$$

$$
\begin{aligned}
& =\exp \left(\sum_{k=1}^{n} a_{k} x_{k}\right)\left[\frac{1}{2}\left[\exp \left(\sum_{k=1}^{n} b_{k} x_{k}\right)+\exp \left(-\sum_{k=1}^{n} b_{k} x_{k}\right)\right]\right]^{p} \\
& =\frac{1}{2^{p}} \exp \left(\sum_{k=1}^{n}\left(a_{k}+p b_{k}\right) x_{k}\right)\left[1+\exp \left(-2 \sum_{k=1}^{n} b_{k} x_{k}\right)\right]^{p} \\
& =\frac{1}{2^{p}} \exp \left(\sum_{k=1}^{n}\left(a_{k}+p b_{k}\right) x_{k}\right) \sum_{m=0}^{\infty} \frac{(p)_{m}}{m !} \exp \left(-2 m \sum_{k=1}^{n} b_{k} x_{k}\right)
\end{aligned}
$$

(Using binomial series)

$$
=\frac{1}{2^{p}} \sum_{m=0}^{\infty} \frac{(p)_{m}}{m !} \exp \left(\sum_{k=1}^{n}\left[a_{k}+(p-2 m) b_{k}\right] x_{k}\right)
$$

Therefore, we obtain the $n$-tuple integral

$$
\begin{array}{r}
\quad \int_{t_{n}}^{r_{n}} \cdots \int_{t_{1}}^{r_{1}} \exp \left(\sum_{k=1}^{n} a_{k} x_{k}\right) \cosh ^{p}\left(\sum_{k=1}^{n} b_{k} x_{k}\right) d x_{1} \cdots d x_{n} \\
=\frac{1}{2^{p}} \cdot \int_{t_{n}}^{r_{n}} \ldots \int_{t_{1}}^{\eta_{1}} \sum_{m=0}^{\infty} \frac{(p)_{m}}{m !} \exp \left(\sum_{k=1}^{n}\left[a_{k}+(p-2 m) b_{k}\right] x_{k}\right) d x_{1} \cdots d x_{n}
\end{array}
$$

$$
=\frac{1}{2^{p}} \cdot \sum_{m=0}^{\infty} \frac{(p)_{m}}{m !} \int_{t_{n}}^{r_{n}} \cdots \int_{t_{1}}^{n} \exp \left(\sum_{k=1}^{n}\left[a_{k}+(p-2 m) b_{k}\right] x_{k}\right) d x_{1} \cdots d x_{n}
$$

(By integration term by term theorem)

$=\frac{1}{2^{p}} \cdot \sum_{m=0}^{\infty} \frac{(p)_{m}}{m !} \prod_{k=1}^{n} \int_{t_{k}}^{r_{k}} \exp \left\{\left[a_{k}+(p-2 m) b_{k}\right] x_{k}\right\} d x_{k}$

$=\frac{1}{2^{p}} \cdot \sum_{m=0}^{\infty} \frac{(p)_{m} \cdot \prod_{k=1}^{n}\left\{\exp \left[a_{k}+(p-2 m) b_{k}\right] r_{k}-\exp \left[a_{k}+(p-2 m) b_{k}\right] t_{k}\right\}}{m ! \prod_{k=1}^{n}\left[a_{k}+(p-2 m) b_{k}\right]}$

Case (B). If $\sum_{k=1}^{n} b_{k} x_{k}<0$, and $\frac{a_{k}-p b_{k}}{-2 b_{k}}$ are not non-negative integers for all $k=1, . ., n$. Because

$$
\exp \left(\sum_{k=1}^{n} a_{k} x_{k}\right) \cosh ^{p}\left(\sum_{k=1}^{n} b_{k} x_{k}\right)
$$

$$
\begin{aligned}
& =\exp \left(\sum_{k=1}^{n} a_{k} x_{k}\right)\left[\frac{1}{2}\left[\exp \left(\sum_{k=1}^{n} b_{k} x_{k}\right)+\exp \left(-\sum_{k=1}^{n} b_{k} x_{k}\right)\right]\right]^{p} \\
& =\frac{1}{2^{p}} \exp \left(\sum_{k=1}^{n}\left(a_{k}-p b_{k}\right) x_{k}\right)\left[1+\exp \left(2 \sum_{k=1}^{n} b_{k} x_{k}\right)\right]^{p} \\
& =\frac{1}{2^{p}} \exp \left(\sum_{k=1}^{n}\left(a_{k}-p b_{k}\right) x_{k}\right) \sum_{m=0}^{\infty} \frac{(p)_{m}}{m !} \exp \left(2 m \sum_{k=1}^{n} b_{k} x_{k}\right)
\end{aligned}
$$

(Using binomial series)

$$
=\frac{1}{2^{p}} \sum_{m=0}^{\infty} \frac{(p)_{m}}{m !} \exp \left(\sum_{k=1}^{n}\left[a_{k}-(p-2 m) b_{k}\right] x_{k}\right)
$$

Thus, the $n$-tuple integral 
$\begin{aligned} & \int_{t_{n}}^{r_{n}} \cdots \int_{t_{1}}^{r_{1}} \exp \left(\sum_{k=1}^{n} a_{k} x_{k}\right) \cosh ^{p}\left(\sum_{k=1}^{n} b_{k} x_{k}\right) d x_{1} \cdots d x_{n} \\ = & \frac{1}{2^{p}} \cdot \int_{t_{n}}^{r_{n}} \cdots \int_{t_{1}}^{n_{1}} \sum_{m=0}^{\infty} \frac{(p)_{m}}{m !} \exp \left(\sum_{k=1}^{n}\left[a_{k}-(p-2 m) b_{k}\right] x_{k}\right) d x_{1} \cdots d x_{n}\end{aligned}$

(Using (6))

$=\frac{1}{2^{p}} \cdot \sum_{m=0}^{\infty} \frac{(p)_{m}}{m !} \int_{t_{n}}^{r_{n}} \cdots \int_{t_{1}}^{n_{1}} \exp \left(\sum_{k=1}^{n}\left[a_{k}-(p-2 m) b_{k}\right] x_{k}\right) d x_{1} \cdots d x_{n}$

(By integration term by term theorem)

$=\frac{1}{2^{p}} \cdot \sum_{m=0}^{\infty} \frac{(p)_{m}}{m !} \prod_{k=1}^{n} \int_{t_{k}}^{r_{k}} \exp \left\{\left[a_{k}-(p-2 m) b_{k}\right] x_{k}\right\} d x_{k}$

$=\frac{1}{2^{p}} \cdot \sum_{m=0}^{\infty} \frac{(p)_{m} \cdot \prod_{k=1}^{n}\left\{\exp \left[a_{k}-(p-2 m) b_{k}\right] r_{k}-\exp \left[a_{k}-(p-2 m) b_{k}\right] t_{k}\right\}}{m ! \cdot \prod_{k=1}^{n}\left[a_{k}-(p-2 m) b_{k}\right]}$

q.e.d.

By Theorem1, we immediately have the following result.

2.5. Corollary 1 Suppose $n$ is any positive integer, $p, a_{k}, b_{k}$ are real numbers for all $k=1, . ., n$.

Case (A). If $b_{k}>0$ and $a_{k}+p b_{k}<0$ for all $k=1, . ., n$. Then the $n$-tuple improper integral

$$
\begin{gathered}
\int_{0}^{\infty} \cdots \int_{0}^{\infty} \exp \left(\sum_{k=1}^{n} a_{k} x_{k}\right) \cosh ^{p}\left(\sum_{k=1}^{n} b_{k} x_{k}\right) d x_{1} \cdots d x_{n} \\
=\frac{(-1)^{n}}{2^{p}} \cdot \sum_{m=0}^{\infty} \frac{(p)_{m}}{m ! \cdot \prod_{k=1}^{n}\left[a_{k}+(p-2 m) b_{k}\right]}
\end{gathered}
$$

Case (B). If $b_{k}<0$ and $a_{k}-p b_{k}<0$ for all $k=1, . ., n$. Then

$$
\begin{gathered}
\int_{0}^{\infty} \cdots \int_{0}^{\infty} \exp \left(\sum_{k=1}^{n} a_{k} x_{k}\right) \cosh ^{p}\left(\sum_{k=1}^{n} b_{k} x_{k}\right) d x_{1} \cdots d x_{n} \\
=\frac{(-1)^{n}}{2^{p}} \cdot \sum_{m=0}^{\infty} \frac{(p)_{m}}{m ! \cdot \prod_{k=1}^{n}\left[a_{k}-(p-2 m) b_{k}\right]}
\end{gathered}
$$

In Corollary 1 , let $x_{k}=\ln y_{k}$, where $y_{k} \geq 1$ for all $k=1, . ., n$. Then we obtain the following result.

2.6. Corollary 2 If the assumptions are the same as Corollary 1.

Case (A). If $b_{k}>0$ and $a_{k}+p b_{k}<0$ for all $k=1, . ., n$. Then the $n$-tuple improper integral

$$
\int_{1}^{\infty} \cdots \int_{1}^{\infty} \prod_{k=1}^{n} y_{k}^{a_{k}-1} \cdot \cosh ^{p}\left(\sum_{k=1}^{n} b_{k} \ln y_{k}\right) d y_{1} \cdots d y_{n}
$$

$$
=\frac{(-1)^{n}}{2^{p}} \cdot \sum_{m=0}^{\infty} \frac{(p)_{m}}{m ! \cdot \prod_{k=1}^{n}\left[a_{k}+(p-2 m) b_{k}\right]}
$$

Case (B). If $b_{k}<0$ and $a_{k}-p b_{k}<0$ for all $k=1, . ., n$. Then

$$
\begin{gathered}
\int_{1}^{\infty} \cdots \int_{1}^{\infty} \prod_{k=1}^{n} y_{k}{ }^{a_{k}-1} \cdot \cosh ^{p}\left(\sum_{k=1}^{n} b_{k} \ln y_{k}\right) d y_{1} \cdots d y_{n} \\
=\frac{(-1)^{n}}{2^{p}} \cdot \sum_{m=0}^{\infty} \frac{(p)_{m}}{m ! \cdot \prod_{k=1}^{n}\left[a_{k}-(p-2 m) b_{k}\right]}(10)
\end{gathered}
$$

The following is the second major result in this paper, we determine the infinite series form of the multiple integral (2).

2.7. Theorem 2 If the assumptions are the same as Theorem 1.

Case (A). If $\sum_{k=1}^{n} b_{k} x_{k}>0$, and $\frac{a_{k}+p b_{k}}{2 b_{k}}$ are not non-negative integers for all $k=1, . ., n$. Then the $n$ -tuple integral

$$
\begin{aligned}
\int_{t_{n}}^{r_{n}} \cdots \int_{t_{1}}^{r_{1}} \exp \left(\sum_{k=1}^{n} a_{k} x_{k}\right) \sinh ^{p}\left(\sum_{k=1}^{n} b_{k} x_{k}\right) d x_{1} \cdots d x_{n} \\
=\frac{1}{2^{p}} \cdot \sum_{m=0}^{\infty} \frac{(-1)^{m}(p)_{m} \cdot \prod_{k=1}^{n}\left\{\exp \left[a_{k}+(p-2 m) b_{k}\right] r_{k}-\exp \left[a_{k}+(p-2 m) b_{k}\right] t_{k}\right\}}{m ! \cdot \prod_{k=1}^{n}\left[a_{k}+(p-2 m) b_{k}\right]}
\end{aligned}
$$

Case (B). If $(-1)^{p}$ exists, $\sum_{k=1}^{n} b_{k} x_{k}<0$, and $\frac{a_{k}-p b_{k}}{-2 b_{k}}$ are not non-negative integers for all $k=1, . ., n$. Then

$$
\begin{aligned}
& \int_{t_{n}}^{r_{n}} \cdots \int_{t_{1}}^{r_{1}} \exp \left(\sum_{k=1}^{n} a_{k} x_{k}\right) \sinh ^{p}\left(\sum_{k=1}^{n} b_{k} x_{k}\right) d x_{1} \cdots d x_{n} \\
= & \frac{(-1)^{p}}{2^{p}} \cdot \sum_{m=0}^{\infty} \frac{(-1)^{m}(p)_{m} \cdot \prod_{k=1}^{n}\left\{\exp \left[a_{k}-(p-2 m) b_{k}\right] r_{k}-\exp \left[a_{k}-(p-2 m) b_{k}\right] t_{k}\right\}}{m ! \cdot \prod_{k=1}^{n}\left[a_{k}-(p-2 m) b_{k}\right]}
\end{aligned}
$$

2.7.1. Proof Case (A). If $\sum_{k=1}^{n} b_{k} x_{k}>0$, and $\frac{a_{k}+p b_{k}}{2 b_{k}}$ are not non-negative integers for all $k=1, . ., n$. Because

$$
\begin{array}{r}
\exp \left(\sum_{k=1}^{n} a_{k} x_{k}\right) \sinh ^{p}\left(\sum_{k=1}^{n} b_{k} x_{k}\right) \\
=\exp \left(\sum_{k=1}^{n} a_{k} x_{k}\right)\left[\frac{1}{2}\left[\exp \left(\sum_{k=1}^{n} b_{k} x_{k}\right)-\exp \left(-\sum_{k=1}^{n} b_{k} x_{k}\right)\right]\right]^{p}
\end{array}
$$




$$
\begin{aligned}
& =\frac{1}{2^{p}} \exp \left(\sum_{k=1}^{n}\left(a_{k}+p b_{k}\right) x_{k}\right)\left[1-\exp \left(-2 \sum_{k=1}^{n} b_{k} x_{k}\right)\right]^{p} \\
& =\frac{1}{2^{p}} \exp \left(\sum_{k=1}^{n}\left(a_{k}+p b_{k}\right) x_{k}\right) \sum_{m=0}^{\infty} \frac{(-1)^{m}(p)_{m}}{m !} \exp \left(-2 m \sum_{k=1}^{n} b_{k} x_{k}\right)
\end{aligned}
$$

(By binomial series)

$$
=\frac{1}{2^{p}} \sum_{m=0}^{\infty} \frac{(-1)^{m}(p)_{m}}{m !} \exp \left(\sum_{k=1}^{n}\left[a_{k}+(p-2 m) b_{k}\right] x_{k}\right)
$$

Therefore, we obtain the $n$-tuple integral

$$
\begin{aligned}
& \int_{t_{n}}^{r_{n}} \cdots \int_{t_{1}}^{r_{1}} \exp \left(\sum_{k=1}^{n} a_{k} x_{k}\right) \sinh ^{p}\left(\sum_{k=1}^{n} b_{k} x_{k}\right) d x_{1} \cdots d x_{n} \\
= & \frac{1}{2^{p}} \cdot \int_{t_{n}}^{r_{n}} \cdots \int_{t_{1}}^{n_{1}} \sum_{m=0}^{\infty} \frac{(-1)^{m}(p)_{m}}{m !} \exp \left(\sum_{k=1}^{n}\left[a_{k}+(p-2 m) b_{k}\right] x_{k}\right) d x_{1} \cdots d x_{n}
\end{aligned}
$$

$=\frac{1}{2^{p}} \cdot \sum_{m=0}^{\infty} \frac{(-1)^{m}(p)_{m}}{m !} \int_{t_{n}}^{r_{n}} \cdots \int_{t_{1}}^{n} \exp \left(\sum_{k=1}^{n}\left[a_{k}+(p-2 m) b_{k}\right] x_{k}\right) d x_{1} \cdots d x_{n}$

(By integration term by term theorem)

$=\frac{1}{2^{p}} \cdot \sum_{m=0}^{\infty} \frac{(-1)^{m}(p)_{m}}{m !} \prod_{k=1}^{n} \int_{t_{k}}^{r_{k}} \exp \left\{\left[a_{k}+(p-2 m) b_{k}\right] x_{k}\right\} d x_{k}$

$=\frac{1}{2^{p}} \cdot \sum_{m=0}^{\infty} \frac{(-1)^{m}(p)_{m} \cdot \prod_{k=1}^{n}\left\{\exp \left[a_{k}+(p-2 m) b_{k}\right] r_{k}-\exp \left[a_{k}+(p-2 m) b_{k}\right] t_{k}\right\}}{m ! \cdot \prod_{k=1}^{n}\left[a_{k}+(p-2 m) b_{k}\right]}$

Case (B). If $(-1)^{p}$ exists, $\sum_{k=1}^{n} b_{k} x_{k}<0$, and $\frac{a_{k}-p b_{k}}{-2 b_{k}}$ are not non-negative integers for all $k=1, . ., n$. Because

$$
\begin{aligned}
& \exp \left(\sum_{k=1}^{n} a_{k} x_{k}\right) \sinh ^{p}\left(\sum_{k=1}^{n} b_{k} x_{k}\right) \\
= & \exp \left(\sum_{k=1}^{n} a_{k} x_{k}\right)\left[\frac{1}{2}\left[\exp \left(\sum_{k=1}^{n} b_{k} x_{k}\right)-\exp \left(-\sum_{k=1}^{n} b_{k} x_{k}\right)\right]\right]^{p} \\
= & \frac{(-1)^{p}}{2^{p}} \exp \left(\sum_{k=1}^{n}\left(a_{k}-p b_{k}\right) x_{k}\right)\left[1-\exp \left(2 \sum_{k=1}^{n} b_{k} x_{k}\right)\right]^{p} \\
= & \frac{(-1)^{p}}{2^{p}} \exp \left(\sum_{k=1}^{n}\left(a_{k}-p b_{k}\right) x_{k}\right) \sum_{m=0}^{\infty} \frac{(-1)^{m}(p)_{m}}{m !} \exp \left(2 m \sum_{k=1}^{n} b_{k} x_{k}\right)
\end{aligned}
$$

(By binomial series)

$=\frac{(-1)^{p}}{2^{p}} \sum_{m=0}^{\infty} \frac{(-1)^{m}(p)_{m}}{m !} \exp \left(\sum_{k=1}^{n}\left[a_{k}-(p-2 m) b_{k}\right] x_{k}\right)$

Thus, we obtain the $n$-tuple integral

$$
\begin{array}{r}
\int_{t_{n}}^{r_{n}} \cdots \int_{t_{1}}^{r_{1}} \exp \left(\sum_{k=1}^{n} a_{k} x_{k}\right) \sinh ^{p}\left(\sum_{k=1}^{n} b_{k} x_{k}\right) d x_{1} \cdots d x_{n} \\
=\frac{(-1)^{p}}{2^{p}} \cdot \int_{t_{n}}^{r_{n}} \cdots \int_{t_{1}}^{r_{1}} \sum_{m=0}^{\infty} \frac{(-1)^{m}(p)_{m}}{m !} \exp \left(\sum_{k=1}^{n}\left[a_{k}-(p-2 m) b_{k}\right] x_{k}\right) d x_{1} \cdots d x_{n}
\end{array}
$$

(Using (14))

$=\frac{(-1)^{p}}{2^{p}} \cdot \sum_{m=0}^{\infty} \frac{(-1)^{m}(p)_{m}}{m !} \int_{t_{n}}^{r_{n}} \cdots \int_{t_{1}}^{n} \exp \left(\sum_{k=1}^{n}\left[a_{k}-(p-2 m) b_{k}\right] x_{k}\right) d x_{1} \cdots d x_{n}$ (By integration term by term theorem)

$$
\begin{aligned}
& =\frac{(-1)^{p}}{2^{p}} \cdot \sum_{m=0}^{\infty} \frac{(-1)^{m}(p)_{m}}{m !} \prod_{k=1}^{n} \int_{t_{k}}^{r_{k}} \exp \left\{\left[a_{k}-(p-2 m) b_{k}\right] x_{k}\right\} d x_{k} \\
& =\frac{(-1)^{p}}{2^{p}} \cdot \sum_{m=0}^{\infty} \frac{(-1)^{m}(p)_{m} \cdot \prod_{k=1}^{n}\left\{\exp \left[a_{k}-(p-2 m) b_{k}\right] r_{k}-\exp \left[a_{k}-(p-2 m) b_{k}\right] t_{k}\right\}}{m ! \cdot \prod_{k=1}^{n}\left[a_{k}-(p-2 m) b_{k}\right]}
\end{aligned}
$$

q.e.d.

By Theorem 2, we obtain the following result.

2.8. Corollary 3Suppose $n$ is any positive integer, $p, a_{k}, b_{k}$ are real numbers.

Case (A). If $b_{k}>0$ and $a_{k}+p b_{k}<0$ for all $k=1, . ., n$. Then the $n$-tuple improper integral

$$
\begin{gathered}
\int_{0}^{\infty} \cdots \int_{0}^{\infty} \exp \left(\sum_{k=1}^{n} a_{k} x_{k}\right) \sinh ^{p}\left(\sum_{k=1}^{n} b_{k} x_{k}\right) d x_{1} \cdots d x_{n} \\
=\frac{(-1)^{n}}{2^{p}} \cdot \sum_{m=0}^{\infty} \frac{(-1)^{m}(p)_{m}}{m ! \cdot \prod_{k=1}^{n}\left[a_{k}+(p-2 m) b_{k}\right]}
\end{gathered}
$$

Case (B). If $(-1)^{p}$ exists, and $b_{k}<0, a_{k}-p b_{k}<0$ for all $k=1, . ., n$. Then

$$
\begin{gathered}
\int_{0}^{\infty} \cdots \int_{0}^{\infty} \exp \left(\sum_{k=1}^{n} a_{k} x_{k}\right) \sinh ^{p}\left(\sum_{k=1}^{n} b_{k} x_{k}\right) d x_{1} \cdots d x_{n} \\
=\frac{(-1)^{n+p}}{2^{p}} \cdot \sum_{m=0}^{\infty} \frac{(-1)^{m}(p)_{m}}{m ! \cdot \prod_{k=1}^{n}\left[a_{k}-(p-2 m) b_{k}\right]}
\end{gathered}
$$

In Corollary 3 , taking $x_{k}=\ln y_{k}$, where $y_{k} \geq 1$ for $k=1, . ., n$. Then the following result holds.

2.9. Corollary 4 If the assumptions are the same as Corollary 3 .

Case (A). If $b_{k}>0$, and $a_{k}+p b_{k}<0$ for all $k=1, . ., n$. Then the $n$-tuple improper integral

$$
\begin{gathered}
\int_{1}^{\infty} \cdots \int_{1}^{\infty} \prod_{k=1}^{n} y_{k}^{a_{k}-1} \cdot \sinh ^{p}\left(\sum_{k=1}^{n} b_{k} \ln y_{k}\right) d y_{1} \cdots d y_{n} \\
=\frac{(-1)^{n}}{2^{p}} \cdot \sum_{m=0}^{\infty} \frac{(-1)^{m}(p)_{m}}{m ! \cdot \prod_{k=1}^{n}\left[a_{k}+(p-2 m) b_{k}\right]}
\end{gathered}
$$

Case (B). If $(-1)^{p}$ exists, $b_{k}<0$, and $a_{k}-p b_{k}<0$ for all $k=1, . ., n$. Then 


$$
\begin{gathered}
\int_{1}^{\infty} \cdots \int_{1}^{\infty} \prod_{k=1}^{n} y_{k}{ }^{a_{k}-1} \cdot \sinh ^{p}\left(\sum_{k=1}^{n} b_{k} \ln y_{k}\right) d y_{1} \cdots d y_{n} \\
=\frac{(-1)^{n+p}}{2^{p}} \cdot \sum_{m=0}^{\infty} \frac{(-1)^{m}(p)_{m}}{m ! \cdot \prod_{k=1}^{n}\left[a_{k}-(p-2 m) b_{k}\right]}
\end{gathered}
$$

\section{Examples}

In the following, for the two types of multiple integrals in this study, we provide some examples and use our theorems and corollaries to determine the infinite series forms of these multiple integrals. On the other hand, we employ Maple to calculate the approximations of these multiple integrals and their solutions for verifying our answers.

3.1. Example 1 Using Case (A) of Theorem 1, we obtain the double integral

$$
\begin{array}{r}
\int_{1}^{3} \int_{2}^{5} \exp \left(-5 x_{1}-4 x_{2}\right) \cosh ^{2 / 3}\left(7 x_{1}+4 x_{2}\right) d x_{1} d x_{2} \\
=\frac{1}{2^{2 / 3}} \cdot \sum_{m=0}^{\infty} \frac{(2 / 3)_{m}}{m !} . \\
\frac{[\exp (-5 / 3-70 m)-\exp (-2 / 3-28 m)][\exp (-4-24 m)-\exp (-4 / 3-8 m)]}{(-1 / 3-14 m)(-4 / 3-8 m)}
\end{array}
$$

We use Maple to verify the correctness of (19) as follows: $>$ evalf(Doubleint $\left(\exp (-5 * \mathrm{x} 1-4 * \mathrm{x} 2) *(\cosh (7 * \mathrm{x} 1+4 * \mathrm{x} 2))^{\wedge}(2 /\right.$ $3), \mathrm{x} 1=2 . .5, \mathrm{x} 2=1 . .3), 18)$;

\subsection{0}

$>\operatorname{evalf}\left(1 / 2^{\wedge}(2 / 3) * \operatorname{sum}(\operatorname{product}(2 / 3-\mathrm{j}, \mathrm{j}=0 . .(\mathrm{m}-1)) / \mathrm{m} ! *(\exp \right.$ $(-5 / 3-70 * \mathrm{~m})-\exp (-2 / 3-28 * \mathrm{~m})) *(\exp (-4-24 * \mathrm{~m})-\exp (-4 / 3-8$ $* \mathrm{~m})) /((-1 / 3-14 * \mathrm{~m}) *(-4 / 3-8 * \mathrm{~m})), \mathrm{m}=0$..infinity $), 18)$;

$$
0.112831641694322300
$$

Next, by Case (B) of Theorem 1, we can determine

$$
\begin{array}{r}
\int_{-2}^{3} \int_{-4}^{-2} \exp \left(-5 x_{1}-4 x_{2}\right) \cosh ^{2 / 3}\left(7 x_{1}+4 x_{2}\right) d x_{1} d x_{2} \\
=\frac{1}{2^{2 / 3}} \cdot \sum_{m=0}^{\infty} \frac{(2 / 3)_{m}}{m !} . \\
\frac{[\exp (58 / 3-28 m)-\exp (116 / 3-56 m)][\exp (-20+24 m)-\exp (40 / 3-16 m)]}{(-29 / 3+14 m)(-20 / 3+8 m)}
\end{array}
$$

Using Maple to verify the correctness of (20) as follows: $>\operatorname{evalf}\left(\right.$ Doubleint $\left(\exp (-5 * x 1-4 * x 2) *(\cosh (7 * x 1+4 * x 2))^{\wedge}(2 /\right.$ 3), $\mathrm{x} 1=-4 . .-2, \mathrm{x} 2=-2 . .3), 22)$;

\section{$3.744905898911145597393 \cdot 10^{20}$}

$>\operatorname{evalf}\left(1 / 2^{\wedge}(2 / 3) * \operatorname{sum}(\operatorname{product}(2 / 3-\mathrm{j}, \mathrm{j}=0 . .(\mathrm{m}-1)) / \mathrm{m} ! *(\exp (58\right.$ $/ 3-28 * \mathrm{~m})-\exp (116 / 3-56 * \mathrm{~m})) *(\exp (-20+24 * \mathrm{~m})-\exp (40 / 3-16$ $* \mathrm{~m})) /((-29 / 3+14 * \mathrm{~m}) *(-20 / 3+8 * \mathrm{~m})), \mathrm{m}=0$..infinity),22);

$$
3.744905898911145597393 \cdot 10^{20}
$$

On the other hand, by Case (A) of Corollary 1, we obtain

$$
\begin{gathered}
\int_{0}^{\infty} \int_{0}^{\infty} \exp \left(-5 x_{1}-4 x_{2}\right) \cosh ^{2 / 3}\left(7 x_{1}+4 x_{2}\right) d x_{1} d x_{2} \\
=\frac{1}{2^{2 / 3}} \cdot \sum_{m=0}^{\infty} \frac{(2 / 3)_{m}}{m !(-1 / 3-14 m)(-4 / 3-8 m)}
\end{gathered}
$$

Verifying the correctness of (21) as follows:

$>$ evalf(Doubleint $\left(\exp (-5 * x 1-4 * x 2) *(\cosh (7 * x 1+4 * x 2))^{\wedge}(2 /\right.$ 3), $\mathrm{x} 1=0$..infinity, $\mathrm{x} 2=0$..infinity));

\subsection{0}

$>\operatorname{evalf}\left(1 / 2^{\wedge}(2 / 3) * \operatorname{sum}(\operatorname{product}(2 / 3-\mathrm{j}, \mathrm{j}=0 . .(\mathrm{m}-1)) /(\mathrm{m} ! *(-1 / 3-1\right.$ $4 * m) *(-4 / 3-8 * m)), m=0$..infinity $)$;

\subsection{1}

3.2. Example 2 By Case (A) of Theorem 2, we can determine the double integral

$$
\begin{array}{r}
\int_{-1}^{2} \int_{-4}^{-2} \exp \left(-3 x_{1}-4 x_{2}\right) \sinh ^{4 / 9}\left(-2 x_{1}-x_{2}\right) d x_{1} d x_{2} \\
=\frac{1}{2^{4 / 9}} \cdot \sum_{m=0}^{\infty} \frac{(-1)^{m}(4 / 9)_{m}}{m !} . \\
\frac{[\exp (70 / 9-8 m)-\exp (140 / 9-16 m)][\exp (-80 / 9+4 m)-\exp (40 / 9-2 m)]}{(-35 / 9+4 m)(-40 / 9+2 m)}
\end{array}
$$

We verify the correctness of (22) using Maple.

$>$ evalf(Doubleint $\left(\exp (-3 * x 1-4 * x 2) *(\sinh (-2 * x 1-x 2))^{\wedge}(4 / 9)\right.$, $\mathrm{x} 1=-4 . .-2, \mathrm{x} 2=-1 . .2), 14)$;

$$
2.0619245987087 \cdot 10^{7}
$$

$>\operatorname{evalf}\left(1 / 2^{\wedge}(4 / 9)^{*} \operatorname{sum}\left((-1)^{\wedge} \mathrm{m}^{*} \operatorname{product}(4 / 9-\mathrm{j}, \mathrm{j}=0 . .(\mathrm{m}-1)) / \mathrm{m}\right.\right.$ ! $*(\exp (70 / 9-8 * \mathrm{~m})-\exp (140 / 9-16 * \mathrm{~m})) *(\exp (-80 / 9+4 * \mathrm{~m})-\exp ($ $40 / 9-2 * \mathrm{~m})) /((-35 / 9+4 * \mathrm{~m}) *(-40 / 9+2 * \mathrm{~m})), \mathrm{m}=0$..infinity $), 14)$;

\section{$2.0619245987086 \cdot 10^{7}$}

In addition, using Case (B) of Theorem 2, we can evaluate

$$
\begin{aligned}
& \int_{-3}^{4} \int_{2}^{6} \exp \left(-3 x_{1}-4 x_{2}\right) \sinh ^{4 / 9}\left(-2 x_{1}-x_{2}\right) d x_{1} d x_{2} \\
& =\frac{1}{2^{4 / 9}} \cdot \sum_{m=0}^{\infty} \frac{(-1)^{m}(4 / 9)_{m}}{m !} . \\
& \frac{[\exp (-38 / 3-24 m)-\exp (-38 / 9-8 m)][\exp (-128 / 9-8 m)-\exp (32 / 3+6 m)]}{(-19 / 9-4 m)(-32 / 9-2 m)}
\end{aligned}
$$

We use Maple to verify the correctness of (23).

$>\operatorname{evalf}\left(\right.$ Doubleint $\left(\exp (-3 * x 1-4 * x 2) *\left((\sinh (-2 * x 1-x 2))^{\wedge} 4\right)^{\wedge}(1\right.$ (9), $\mathrm{x} 1=2 . .6, \times 2=-3 . .4), 14)$;

\subsection{3}

$>\operatorname{evalf}\left(1 / 2^{\wedge}(4 / 9) * \operatorname{sum}\left((-1)^{\wedge} \mathrm{m}^{*} \operatorname{product}(4 / 9-\mathrm{j}, \mathrm{j}=0 . .(\mathrm{m}-1)) / \mathrm{m}\right.\right.$ ! $*(\exp (-38 / 3-24 * \mathrm{~m})-\exp (-38 / 9-8 * \mathrm{~m})) *(\exp (-128 / 9-8 * \mathrm{~m})-\exp$ $(32 / 3+6 * \mathrm{~m})) /((-19 / 9-4 * \mathrm{~m}) *(-32 / 9-2 * \mathrm{~m})), \mathrm{m}=0$..infinity $), 14)$;

\subsection{5}

On the other hand, by Case (B) of Corollary 3, we have 


$$
\begin{gathered}
\int_{0}^{\infty} \int_{0}^{\infty} \exp \left(-3 x_{1}-4 x_{2}\right) \sinh ^{4 / 9}\left(-2 x_{1}-x_{2}\right) d x_{1} d x_{2} \\
\quad=\frac{1}{2^{4 / 9}} \cdot \sum_{m=0}^{\infty} \frac{(-1)^{m}(4 / 9)_{m}}{m !(-19 / 9-4 m)(-32 / 9-2 m)}
\end{gathered}
$$

Using Maple to verify the correctness of (24).

$>\operatorname{evalf}\left(\right.$ Doubleint $\left(\exp (-3 * x 1-4 * x 2) *\left((\sinh (-2 * x 1-x 2))^{\wedge} 4\right)^{\wedge}(1\right.$ 19), $\mathrm{x} 1=0$..infinity, $\mathrm{x} 2=0$..infinity), 14$)$;

\subsection{8}

$>\operatorname{evalf}\left(1 / 2^{\wedge}(4 / 9) * \operatorname{sum}\left((-1)^{\wedge} \mathrm{m}^{*} \operatorname{product}(4 / 9-\mathrm{j}, \mathrm{j}=0 . .(\mathrm{m}-1)) /(\mathrm{m}\right.\right.$ ! $*(-19 / 9-4 * m) *(-32 / 9-2 * m)), m=0$..infinity), 14$)$;

$$
0.086402438262562
$$

\section{Conclusions}

As mentioned, the multiple integral problem is important in probability theory and quantum field theory. In this study, we propose a new technique to evaluate two types of multiple integrals, and we hope this method can be applied in mathematical statistics or quantum physics. On the other hand, we know the binomial series and the integration term by term theorem play significant roles in the theoretical inferences of this study. In fact, the applications of these two theorems are extensive, and can be used to easily solve many difficult problems; we endeavor to conduct further studies on related applications. In addition, Maple also plays a vital assistive role in problem-solving. In the future, we will extend the research topic to other calculus and engineering mathematics problems and solve these problems by using Maple. These results will be used as teaching materials for Maple on education and research to enhance the connotations of calculus and engineering mathematics.

\section{REFERENCES}

[1] R. J. Stroeker and J. F. Kaashoek, Discovering Mathematics with Maple : An Interactive Exploration for Mathematicians, Engineers and Econometricians, Basel: Birkhauser Verlag, 1999.

[2] M. L. Abell and J. P. Braselton, Maple by Example, 3rd ed., New York: Elsevier Academic Press, 2005.

[3] C. T. J. Dodson and E. A. Gonzalez, Experiments in Mathematics Using Maple, New York: Springer-Verlag, 1995.

[4] J. S. Robertson, Engineering Mathematics with Maple, New York: McGraw-Hill, 1996.

[5] D. Richards, Advanced Mathematical Methods with Maple, New York: Cambridge University Press, 2002.

[6] F. Garvan, The Maple Book, London: Chapman \& Hall/CRC, 2001.
[7] C. Tocci and S. G. Adams, Applied Maple for Engineers and Scientists, Boston: Artech House, 1996.

[8] F. Streit,On multiple integral geometric integrals and their applications to probability theory, Canadian Journal of Mathematics, Vol. 22, pp. 151-163, 1970.

[9] L. H. Ryder, Quantum Field Theory, 2nd ed., New York: Cambridge University Press, 1996.

[10] C. -H. Yu, The evaluation of two types of multiple improper integrals, Proceedings of 2012 Chang Yun Ka alliance of colleges Symposium, Taiwan, M-7, 2012.

[11] C. -H. Yu, Application of Maple: multiple integrals as examples, Proceedings of the 6th Cross-Strait Technology and Humanities Education and University-Industry Cooperation Seminar, Taiwan, EMD001, 2012.

[12] C.-H. Yu, A study on the multiple improper integral problems with Maple, Applied Mechanics and Materials, in press.

[13] C. -H. Yu, Using Maple to study the multiple improper integral problem, Proceedings of IIE Asian Conference 2013, Taiwan, Vol. 1, pp. 625-632, 2013.

[14] C. -H. Yu, Application of Maple on evaluating multiple improper integrals, Proceedings of the 6th IEEE/International Conference on Advanced Infocomm Technology, Taiwan, No. 00282, 2013.

[15] C. -H. Yu, Application of Maple: the evaluation of double integral as an example, Proceedings of 2013 International Symposium on Intercultural Communication, Taiwan, pp. 294-302, 2013.

[16] C. -H. Yu, Application of Maple on evaluating the double improper integrals, Proceedings of the Innovative Education and Learning Technology, 2013.

[17] C. -H. Yu, Application of Maple: double improper integrals as examples, Proceedings of 2013 Information Education and Technology Application Conference, Taiwan, pp. 1-5, 2013.

[18] C. -H. Yu, Using Maple to study multiple improper integrals, International Journal of Research in Information Technology, Vol. 1, Issue. 8, pp. 10-14, 2013.

[19] C. -H. Yu,A study on the multiple improper integral problems, Journal of Hsin Sheng, in press.

[20] C. -H. Yu, Application of Maple: taking the evaluation of double improper integrals as an example, Proceedings of the 7th International Conference on Information Technology, Taiwan, No.28, 2013.

[21] C. -H. Yu,Application of Maple: two types of improper integrals as examples, Proceedings of 2013 Information Technology and Management Symposium, Taiwan, B9-B14, 2013.

[22] C. -H. Yu,Application of Maple onthe evaluation of four types of double integrals, Proceedings of 2013 Business Innovation and Development Conference, Taiwan, B20130117002, 2013.

[23] C. -H. Yu,Application of Mapleon solving some type of multiple integrals, Proceedings of the 8th Information Management and Practice Conference, Taiwan, TP20120128, 2012.

[24] R. Courant and F. John, Introduction to Calculus and 
Analysis, Vol. 1,New York: Springer-Verlag, pp.456-457, 1989.

[25] T. M. Apostol, Mathematical Analysis, 2nd ed., Boston: Addison-Wesley, p269, 1975. 